\title{
Satiation in name and face recognition
}

\author{
MICHAEL B. LEWIS and HADYN D. ELLIS \\ Cardiff University, Cardiff, Wales
}

\begin{abstract}
Massive repetition of a word can lead to a loss of meaning (i.e., semantic satiation). Satiation is a general property of neurons and so it would be expected that semantic satiation would be found for stimuli other than words. Three experiments examined the occurrence of satiation for the repetition of names, the visualization of faces, and the repetition of faces. Reaction times to a decision based on a repeated name or face were longer following 30 repetitions than following 3 repetitions, indicating that satiation had occurred. Mere visualization over the same time interval, however, did not elicit satiation effects.
\end{abstract}

Semantic satiation refers to a temporary loss of meaning for a word that has either been massively repeated or fixated on for an extended period (see Esposito \& Pelton, 1971, for an extensive review of early work). Smith (1984) demonstrated the importance of meaning in this phenomenon. He found a semantic satiation effect in a category membership task but not in a lexical decision task. In the category membership experiment, participants read a category name either 3 times (control) or 30 times (satiation). They then had to decide whether or not a word was a member of that category. Participants were faster to make category decisions following 3 repetitions than following 30 repetitions; from this result it was inferred that the category name was more satiated over the 30 repetitions. The failure to find semantic satiation in lexical decision tasks can be explained by assuming that these tasks do not require access to the meaning of the repeated word (see Smith \& Klein, 1990).

The great majority of experiments studying semantic satiation have used repeated words. There are many aspects of behavior, however, that exhibit satiation, including the most basic of behaviors (e.g., feeding and mating). Satiation, or fatigue, is also a basic property of neural activity: A period of sustained activation will be followed by a reduction in sensitivity (e.g., Forbes \& Rice, 1929; Gerard \& Forbes, 1928). If this is the case, we would expect to see forms of satiation in other cognitive processes as well. The experiments reported here examined the possibility of satiation effects caused by the repetition of celebrities' names and the repetition of their faces.

Evidence for the presence of satiation effects with such stimuli would have particular ramifications on accounts of how names and faces are stored and retrieved. Computation models for face and name recognition (e.g., Burton, Bruce, \& Johnston, 1990, and Burton, 1994, or Farah, O'Reilly, \& Vecera, 1993), however, do not offer accounts for satiation effects. This is not surprising given that satia-

We thank two anonymous reviewers for their constructive comments on an earlier version. Correspondence should be addressed to $\mathrm{M}$. B. Lewis, School of Psychology, Cardiff University, P. O. Box 901, Cardiff CF1 3YG,Wales (e-mail: lewismb@cardiff.ac.uk). tion effects have never been found with such stimuli (previous experiments that explored the issue failed to find clear satiation effects, e.g., Lewis \& Ellis, 1999, in press). Consequently, the following experiments were aimed at identifying satiation effects with name and face stimuli.

\section{EXPERIMENT 1}

The goal of the first two experiments was to extend the effects of satiation seen with words to the domain of the massive repetition or prolonged fixation of names. It can be argued that names are like words in many ways - indeed, they are presented and read as words. In spite of these similarities, the examination of satiation with names is not a trivial generalization from satiation with words. Names differ from words in that they refer to a specific identity rather than to a class of items. This difference was reflected in the nature of the task in the experiment below.

The design of the experiment was based on Smith's (1984) study, in which category names are repeated either 3 or 30 times before a category membership decision has to be made to a word. Reaction time and accuracy to the decision formed the measure of satiation, with longer times for more repetitions being taken as indicative of greater satiation. In Experiment 1, a celebrity's name was read either 3 or 30 times, and the measure of satiation was derived from how quickly the participant identified whether a presented face was the same as the name or of a different person. If satiation was occurring for the celebrity's name over 30 repetitions, its meaning would be lost, and, therefore, the decision that the face was the same as the repeated name would take longer than after only 3 repetitions.

This first experiment investigated the effect of name repetition on subsequent face-to-name matching. If the repetition of the name produces satiation then it is expected to see an increase in the reaction times as the number of repetitions increases.

\section{Method \\ Participants. Thirty-six undergraduates participated in the study as part of their course requirements. All had normal or corrected-} to-normal vision. 
Stimuli. The stimuli consisted of 24 color faces of famous people together with 24 written names. The names given to each face were the modal names provided by 10 judges who did not participate in the experiment. Some names used were the persons' real names (e.g., Tony Blair), some names were the persons' nicknames (e.g., Ginger Spice was given more often than Geri Halliwell, previously one of the Spice Girls), and some names were of the character most played by an actor (e.g., Bianca Butcher-a character in a popular U.K. TV program--was given more often than Patsy Palmer-the actress's name). The 24 faces were chosen from a set of 36 faces seen by the judges. The two criteria for selection were that all 10 judges had to recognize the person, and the faces that produced most uniformity of names were selected (all of the experimental stimuli were given consistent names by at least 8 out of the 10 judges).

The faces and names were presented using an Apple Multiscan 15 display. The faces were presented in color and subtended a visual angle of $9^{\circ}$ in the vertical direction. The names were presented in upper- and lowercase "Times" font with capital letters subtending a visual angle of $2^{\circ}$ in the vertical direction.

Procedure. Participants sat in front of a computer and were presented with a series of 24 trials. Each trial began with the presentation of a celebrity name that was repeated either 3 times or 30 times. The name was presented for 500 msec each time and there was an interval of $200 \mathrm{msec}$ between each presentation. The participant was required to read the name aloud every time it appeared. Immediately following the presentations of the name for the last time, a cross was presented for $500 \mathrm{msec}$ to provide a cue for the fact that a face was about to follow. A face was presented after the cross, and the participant was required to indicate whether the face was that of the same person or a different person to the name they had been reading. The face remained on the screen until a response was made using one of two keys. There was then a break of $5 \mathrm{sec}$ before the next trial started.

The procedure began with four practice trials. The 20 experimental trials were in four different conditions as determined by two factorial variables: Half of the trials used 3 repetitions whereas the other half used 30 , but also, half of the trials used the same name and face (and so required a same response), whereas the other half used different names and faces (and so required a different response). The faces that occurred in each of the four within-participants conditions were counterbalanced between participants. The order of the experimental trials was randomized for each participant

Design. The dependent variable was the time taken, from the onset of the face, to indicate correctly whether or not it was the same person as the previous name. The two factorial independent variables were the number of times the name had been read and whether the face belonged to or was different from the name.

\section{Results and Discussion}

Errors made up less than $1 \%$ of responses, and these were removed from the analysis. The mean reaction times for the four conditions are shown in Figure 1. The reaction times following 3 repetitions were faster than the reaction times following 30 repetitions. This was true for both same and different judgments, but the difference was larger for same judgments.

Two-way analyses of variance (ANOVAs) were conducted on the reaction time data by participants and by items. The factors of the ANOVA were number of repetitions and whether the target was the same as or different from the repeated name. These factors were both within participant and within items. There was a significant effect of number of repetitions $\left[F_{p}(1,35)=15.90, F_{i}(1,19)=\right.$ $7.72, p<.05]$. The effect of whether or not the face was the same as the name was nonsignificant $\left[F_{p}(1,35)=2.04\right.$, $\left.F_{i}(1,19)<1, p>.05\right]$, but the interaction was significant by participants $\left[F_{p}(1,35)=5.05, p<.05, F_{i}(1,19)=1.53\right.$, $p>.05]$. Analysis of simple main effects revealed a significant effect of number of repetitions when the name and face were the same $\left[F_{p}(1,35)=16.89, F_{i}(1,19)=\right.$ $4.70, p<.001]$, but this effect was not significant when the name and face were different $\left[F_{p}(1,35)=3.21\right.$, $\left.F_{i}(1,19)<1, p>.05\right]$.

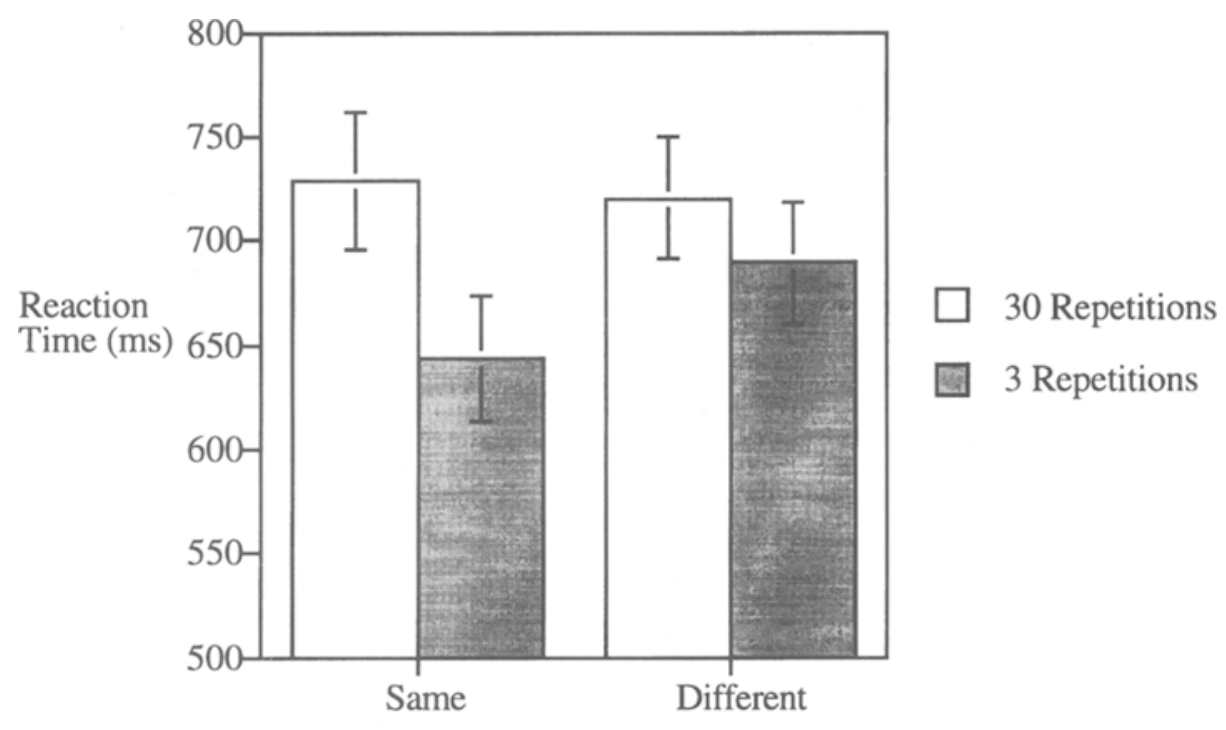

Condition

Figure 1. Mean reaction times for the four conditions in Experiment 1. The error bars show standard errors. 
The only reliable result found was that the participants were slower to make the same decisions (i.e., the face was the same as the name) after 30 repetitions of the name than after 3 repetitions (this difference was not significant for the cases when the name and faces were different). This supports the hypothesis that massive repetition of a person's name can lead to semantic satiation, or loss of meaning.

\section{EXPERIMENT 2}

While Experiment 1 indicated that satiation effects can be found in name repetition, it did not determine whether the effect is very different from satiation seen with words. Even though the test items were faces, the locus of satiation may have been the name's phonology. It is possible that words and names are satiated following massive repetition of their phonology (either by vocalization or by auditory presentation, e.g., Pilotti, Antrobus, \& Duff, 1997), and so it may be the phonology that is central to semantic satiation. Alternatively, satiation may be a general property of neural systems as a consequence of attenuation or habituation. If this is the case, one would expect to find satiation effects for nonverbal stimuli.

The idea of satiation without vocalization is not a new one. Indeed, Severance and Washburn (1907) demonstrated semantic satiation with prolonged fixation. More recently, Esposito (1987) reported an experiment in which a word was fixated on for either $3 \mathrm{~min}$ or $2 \mathrm{sec}$. Although Esposito's study demonstrated the possibility of perceptual satiation, it only found a nonsignificant trend for the existence of semantic satiation in the same task. The question of whether it is possible to find satiation without any phonological activity (either auditory or vocalization) is addressed in Experiment 2. The method employed in this experiment was similar to that used in Experiment $1 \mathrm{ex}$ cept that participants did not read the name of the celebrity aloud. Instead, the participants were required to visualize the face of the celebrity for the duration of presentation. This method was employed because it was thought that visualization would still lead to activation, but without the verbal production of the name, as used in the first experiment. If the same latency increases were found in this experiment as well as in Experiment 1, then it could be concluded that phonological activity is not a prerequisite of satiation to occur but that it is a more general property of cognitive systems.

There is strong evidence that people vary in their abilities to visualize objects. In order to investigate whether these individual differences affect the size of satiation effects in Experiment 2, the Vividness of Visualization Imagery Questionnaire (VVIQ), developed by Marks (1973), was administered prior to the satiation procedure. From this questionnaire it is possible to attribute a number to each participant indicating how easily he/she can visualize objects (a low number indicates a good visualizer).
This experiment examined whether a satiation effect for names would occur following visualization of a person's face, together with the constant presentation of the name. If visualization does produce satiation, this will be reflected in an increase in reaction times for the face-toname matching for longer visualization times.

\section{Method}

Participants. Thirty-six undergraduates participated in the study as part of a course requirement, none of whom had participated in Experiment 1. All had normal or corrected-to-normal vision.

Stimuli. The stimuli consisted of the same 24 color faces of famous people, together with the same 24 written names used in Experiment 1.

Procedure. The experiment comprised two sections. In the first section, participants completed Marks's (1973) VVIQ. This questionnaire was used to determine each participant's ability to visualize.

The second section of the experiment was similar to Experiment 1 , but with a number of key differences. In this version of the experiment the names were presented continuously for the same length of time as they had been shown in Experiment 1 (i.e., a total of 2.1 or $21 \mathrm{sec}$ ). During the presentation of the name, the participants did not read it aloud, but were instructed to visualize that person. As in Experiment 1, the participants were then presented with a face that was either the same as the name that had been presented or different, and their task was to indicate whether it was the same or different. The practice trials and the use of counterbalancing were the same as in Experiment 1.

Design. The dependent variable was the time taken to indicate correctly whether or not the face was that of the same person as the previous name. The two factorial independent variables were the length of time for which the person had been visualized and whether the face was the same as or different from the name. Participants' rating on the VVIQ were also incorporated into the analysis.

\section{Results and Discussion}

Errors made up less than $1 \%$ of responses and were removed from the analysis. The mean reaction times for the four conditions are shown in Figure 2 . The reaction times following $2.1 \mathrm{sec}$ of visualizations were slightly faster than the reaction times following $21 \mathrm{sec}$ of visualizations. This trend was true for both same and different judgments, but it was larger for same judgments. The size of the reaction time differences between long and short presentations did not correlate with the participants' scores on the VVIQ [ $r(35)=.067]$.

A two-way ANOVA was conducted on the reaction times with factors of length of time of visualization and whether the target was the same as or different from the repeated name. This analysis revealed a significant effect of target type [same responses were faster than different responses, $\left.F_{p}(1,35)=35.33, F_{i}(1,19)=7.91, p<.05\right]$, but the effects of length of visualization and interaction were not significant $\left[F_{p}(1,35)=3.56, F_{i}(1,19)=.41, p>\right.$ .05 , and $F_{p}(1,35)<1, F_{i}(1,19)<1$, respectively]. Analysis of simple main effects demonstrated that the effect of length of presentation was not significant for either same decisions $\left[F_{p}(1,35)=1.24, F_{i}(1,19)<1, p>.05\right]$ or different decisions $\left[F_{p}(1,35)=2.93, F_{i}(1,19)<1, p>.05\right]$. 


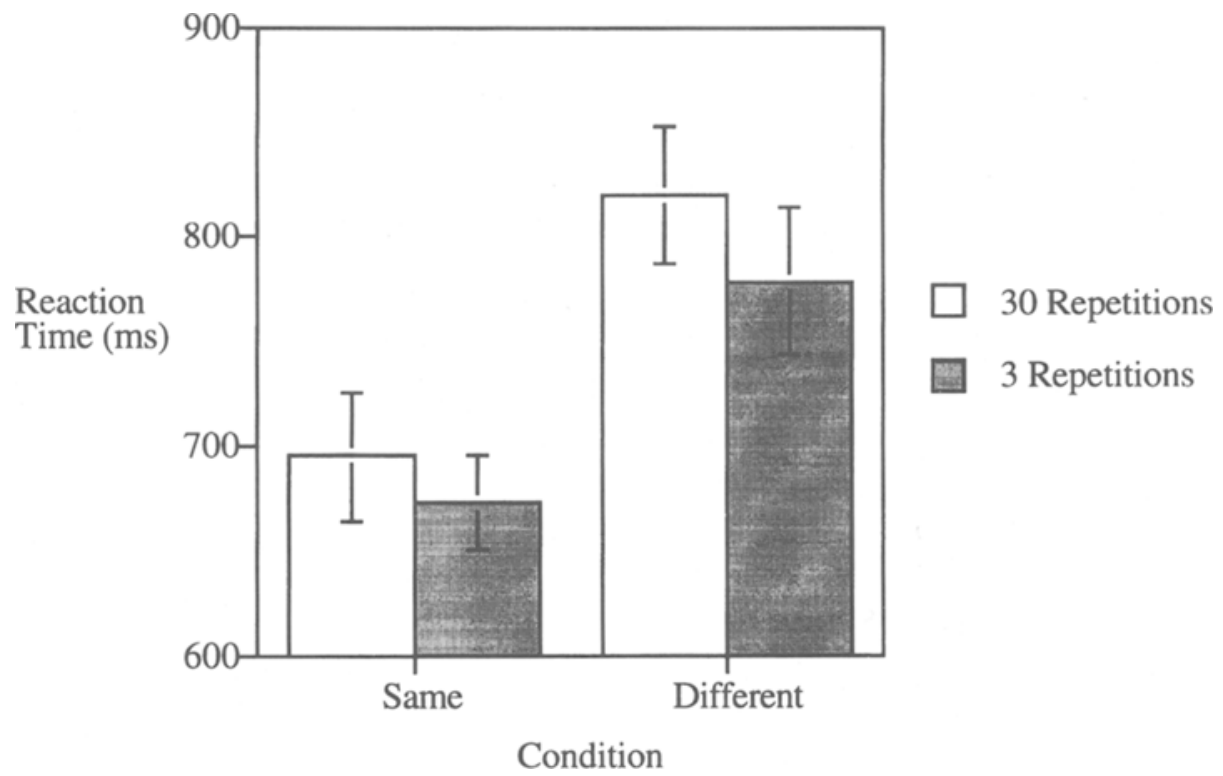

Figure 2. Mean reaction times for the four conditions in Experiment 2. The error bars show standard errors.

The design of Experiment 2 was similar to that employed for Experiment 1, and the same stimuli were used in each. This allowed us to conduct a single ANOVA over both sets of data with the two different experiments being a between-participants variable. The three-way interaction of this ANOVA (experiment*same/different*satiation/ control) approached significance $[F(1,70)=3.65, p=$ $.06]$, and so careful consideration of the simple main effect was required. It was found that the interaction between experiment and presentation time (satiation/control) was significant for the same decisions $[F(1,70)=4.86, p<$ $.05]$ but not for the different decisions $[F(1,70)=0.13]$. This implies that the satiation effect (on same decisions) was larger for Experiment 1 than Experiment 2, suggesting that, perhaps, it is the verbalization of the names that produced a significant satiation effect.

The experiment showed that there was no significant difference in reaction times between the same decision following longer presentation of the names than for the same decision following shorter presentations of the name, although there was a trend in the expected direction. The size of this trend was significantly smaller than the effect observed in Experiment 1 between 30 and 3 repetitions. This experiment thus failed to demonstrate satiation effects for the visualization of a face.

The analysis conducted over both experiments revealed a significant interaction between experiment and condition (satiation or control) when the same decisions were made. The implications of this finding are that repetitive verbalization (as used in Experiment 1) produces greater satiation (for the same decisions) than is produced from observing a name and visualizing the face. Therefore, the satiation observed in Experiment 1 was not merely a feature of the time, since the name was first presented.

\section{EXPERIMENT 3}

Experiment 2 failed to demonstrate a nonverbal satiation effect; therefore, prolonged visualization itself does not appear to be sufficient to produce significant loss of meaning. Although visualization failed to demonstrate a significant satiation effect, this does not necessarily mean that satiation is limited to the verbal domain. Nonverbal satiation is further investigated in the following experiment. For this experiment, faces are used as the satiating stimuli. It was predicted that massive repetitions of different views of the same celebrity would repeatedly activate a person representation, leading to satiation. This satiation would make access to semantic information about that person more difficult because it is necessary to activate the satiated, or fatigued, representation in order to retrieve semantic information.

The test of whether satiation has occurred involved repeatedly presenting the face of one celebrity and asking the participant to decide whether or not another celebrity was related to the target person. This task was chosen because it requires access to the semantic information associated with the repeated face (which is considered to be the locus for the satiation effects). As in Experiment 1, the satiation contrast was either 3 repetitions (control) or 30 repetitions (satiation) of the item. If the repeated face loses its meaning over the 30 repetitions, it will be more difficult for participants to make the judgment that another celebrity is either related or unrelated to the first. 


\section{Method}

Participants. Twenty undergraduates received payment for their participation in the study, none of whom had taken part in Experiment 1 or 2. All had normal or corrected-to-normal vision.

Stimuli. The experimental stimuli consisted of images of the faces of 40 famous people selected so that they could be placed into associate pairs of related people. Ratings for the semantic relatedness of each associate pair and each face paired with a different person from the set (i.e., the pairs used in the unrelated test condition described below were rated) were obtained from six judges who did not participate in the experiment. The judges rated each pair on a 7 point scale $(1=$ highly associated, $7=$ not associated $)$. The ratings of the associate pairs $(2.10, S D=0.61)$ were lower than those of the nonassociated pairs $(5.54, S D=0.77)$, and this difference was found to be highly significant $[t(39)=15.47, p<.01]$. There were four additional practice faces that consisted of an associated pair and a nonassociated pair.

The faces were presented using an Apple Multiscan 15 display. Each face was presented in color and subtended a visual angle of $5^{\circ}$ in the vertical direction.

Procedure. Each participant was presented with a series of 22 trials, the first 2 of which were practice trials. During each trial, the participant was presented with a series of different views of the same famous person. This series was either 30 pictures long (for the satiation condition) or 3 pictures long (for the control condition), and the images were presented at a rate of 1 per second (the face was presented for $900 \mathrm{msec}$ followed by an interval of $100 \mathrm{msec}$ ). Immediately after the series, a cross was presented for $1 \mathrm{sec}$ as a cue for the test face that followed. The test face was either the face of a semantically related person (an associate) or a semantically unrelated person (a nonassociate), and each participant's task was to decide whether they were related and to press a key indicating his/her decision.

The series of trials began with the practice items. Over the 20 experimental trials, there were 5 trials for each of the four factorial conditions on association (related or unrelated) and satiation ( 3 or 30 repetitions). The order of the experimental trials was randomized, and which faces occurred in which of the four conditions was counterbalanced across participants.

Design. The dependent variable was the reaction times to make relatedness decisions to the target faces. The independent variables were the number of repetitions and whether the target faces were related or unrelated to the cue faces.

\section{Results and Discussion}

Errors made up less than $5 \%$ of responses, and reaction times from these were removed from the analysis. The mean reaction times for the four conditions are shown in Figure 3. When the items were associated, the reaction times following 3 repetitions were faster than the reaction times following 30 repetitions.

Two-way ANOVAs were conducted on the reaction time data by participants and by items. There were withinparticipant and within-item factors of number of repetitions ( 30 or 3 ) and relatedness (related or unrelated test faces). These analyses revealed a significant main effect of number of repetitions $\left[F_{p}(1,19)=5.62, p<.05\right.$, $\left.F_{i}(1,19)=9.29, p<.05\right]$. The effect of relatedness was significant $\left[F_{p}(1,19)=8.83, p<.05, F_{i}(1,19)=4.71, p<\right.$ $.05]$. The interaction was not significant $\left[F_{p}(1,19)=\right.$ $\left.1.84, p>.05, F_{i}(1,19)<1, p>.05\right]$. Analysis of the simple main effect revealed that the number-of-repetitions effect was significant for the related test faces $\left[F_{p}(1,19)=\right.$ $\left.4.52, p<.05, F_{i}(1,19)=5.53, p<.05\right]$ but not for unrelated test faces $\left[F_{p}(1,19)=3.31, p>.05, F_{i}(1,19)=\right.$ $0.21, p>.05]$.

Experiment 3 clearly shows an effect of satiation, with association decisions following 30 repetitions of a face taking longer than those following 3 repetitions. That is, massive repetition of different views of a face seems to impair processing in such a way that when the participant attempted to retrieve semantic information (i.e., was the target related to the repeated face), this information was more difficult to obtain.

The result of this experiment implies that it is not the repetition of the phonology that leads to the satiation in

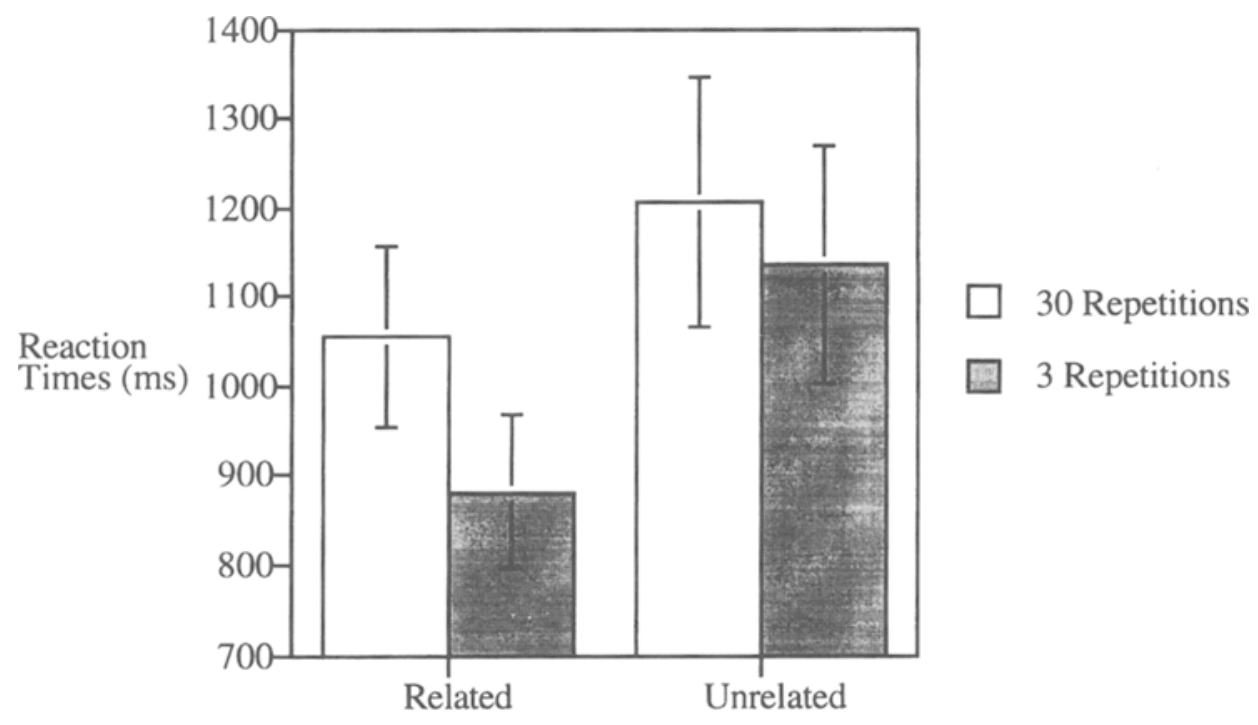

Condition

Figure 3. Mean reaction times for the four conditions in Experiment 3. The error bars show standard errors. 
experiments. Rather, it would appear that visual repetition of a face per se can produce a satiation effect.

\section{GENERAL DISCUSSION}

The results of the three experiments, taken together, have a number of ramifications. Specifically, they have implications for the way we model the processes of face recognition. Experiments 1 and 3 demonstrate that satiation effects can be found in name and face recognition, respectively. In Experiment 1, the massive repetition of a name made more difficult a subsequent decision as to whether a face was that person's. In Experiment 3, a similar massive repetition of a face again made subsequent decisions as to whether a second face was semantically related to the first more difficult. The former experiment is similar to displays of semantic satiation using massive repetition of words. The latter experiment shows that the effects of satiation are also characteristic of nonlexical stimuli and can be found with the repetition of many faces.

The results of the experiments reported here mean that there is a new set of data that must be accounted for in any complete model of face and name processing. Computation models such as Burton's (1994) and Farah et al.'s (1993) do not display satiation. It may be possible to modify either or both of these models so that they can account for satiation effects. In the light of the current debate surrounding these two conflicting models (see Young \& Burton, 1999; O'Reilly \& Farah, 1999), satiation may prove to be a useful tool for exploring the two divergent accounts.

\section{REFERENCES}

BURTON, A. M. (1994). Learning new faces in an interactive activation and competition model. Visual Cognition, 1, 313-348.

Burton, A. M., Bruce, V., \& Johnston, R. A. (1990). Understanding face recognition with an interactive activation model. British Journal of Psychology, 81, 361-380.

EsPosito, N. J. (1987). Semantic satiation as a perceptual phenomenon. Perceptual \& Motor Skills, 64, 487-491.

Esposito, N. J., \& Pelton, L. H. (1971). Review of the measurement of semantic satiation. Psychological Bulletin, 75, 330-346.

Farah, M. J., O'ReIlly, R. C., \& Vecera, S. P. (1993). Dissociated overt and covert recognition as an emergent property of a lesioned neural network. Psychological Review, 100, 571-588.

ForBES, A., \& Rice, L. H. (1929). Quantitative studies of nerve impulses IV: Fatigue in peripheral nerve. American Journal of Physiology, 90, 119-145.

Gerard, R. W., \& Forbes, A. (1928). "Fatigue" of the flexion reflex. American Journal of Physiology, 86, 186-205.

LEwIS, M. B., \& ELLIS, H. D. (1999). Repeated repetition priming in face recognition. Quarterly Journal of Experimental Psychology, 52A, 927-955.

LEWIS, M. B., \& ELLIS, H. D. (in press). The effects of massive repetition on speeded recognition of faces. Quarterly Journal of Experimental Psychology.

MARKs, D. F. (1973). Visual imagery differences in the recall of pictures. British Journal of Psychology, 64, 17-24.

O'ReILly, R. C., \& FARAH, M. J. (1999). Simulation and explanation in neuropsychology and beyond. Cognitive Neuropsychology, 16, 49-72.

Pilotti, M., Antrobus, J. S., \& Duff, M. (1997). The effect of presemantic acoustic adaptation on semantic "satiation." Memory \& Cog. nition, 25, 305-312.

Severance, E., \& Washburn, M. F. (1907). The loss of associative power in words after long fixation. American Journal of Psychology, 18, $182-186$

SMITH, L. C. (1984). Semantic satiation affects category membership decision time but not lexical priming. Memory \& Cognition, 12, 483-488.

SMITH, L. [C.], \& KLEIN, R. (1990). Evidence for semantic satiation: Repeating a category slows subsequent semantic processing. Journal of Experimental Psychology: Learning, Memory, \& Cognition, 16, 852-861.

Young, A. W., \& Burton, A. M. (1999). Simulation face recognition: Implications for modelling cognition. Cognitive Neuropsychology, $16,1-48$.

(Manuscript received December 18, 1998; revision accepted for publication September 9,1999 .) 